\title{
Effect of Immature Green Tomato Fruit Color on Yellow Shoulder Incidence and Soluble Solids Content of Ripe Fruit
}

\author{
Matthew R. Mattia and John W. Scott ${ }^{1}$ \\ Gulf Coast Research and Education Center, Institute of Food and Agricultural Sciences, University of \\ Florida, 14625 CR 672, Wimauma, FL 33598-6101
}

\begin{abstract}
ADDITIONAL INDEX wORDS. apple green shoulder, green shoulder, persistent green shoulder, Solanum lycopersicum, uniform green shoulder, yellow top

ABstract. Tomato (Solanum lycopersicum) breeders have observed that plants with uniform green-shouldered fruit are less prone to yellow shoulder (YS) than are plants with (dark) green-shouldered tomatoes and thus have selected for tomato cultivars with uniform green-shouldered fruit. However, a recent publication reported that a cultivar with green-shouldered fruit had significantly higher soluble solids content (SSC) than an isogenic cultivar with uniform green-shouldered fruit and postulated that selection of uniform green shoulder has negatively affected tomato flavor and processing quality. Lines with dark green $\left(u^{+}\right)$, uniform green $(u)$, uniform gray-green $(u g)$, apple green $\left(u^{\mathrm{Ag}}\right)$, medium green, and pale green $\left(u^{\mathrm{Pg}}\right)$ immature fruit colors were crossed in all combinations to produce $F_{1}$ plants that were self-pollinated to produce $F_{2}$ seed. Parents, $F_{1}$, and $F_{2} s$ were planted in the field in completely randomized block designs over two seasons. Plants were visually phenotyped for immature fruit color, and fruit from each plant were selected to measure shoulder and base color with a colorimeter. Ripening fruit were harvested to measure the incidence of YS, and SSC was measured on ripe fruit from each plant with a refractometer. In the spring season, fruit from $F_{2}$ plants with green-shouldered fruit had significantly higher YS incidence than all phenotypes with uniform fruit colors. In the fall, phenotypes with medium-green shoulders were also tested, and these had greater YS than all other phenotypes except green shoulder. YS incidence for green shoulder was not significantly greater than that in the other phenotypes. Fla. 7956, the apple green parent, had 0\% YS and appeared to be resistant. Higher SSC was observed in the spring season than in the fall season. However, in both seasons, when comparisons were made between phenotypes that segregated in the $\mathrm{F}_{2} \mathrm{~s}$, the SSC of green-shouldered phenotypes was not significantly higher than that of other phenotypes. Plants with apple green fruit tended to be higher in SSC in the fall, but this may relate to the dark green foliage of apple green plants and not just the fruit color. A hypothesis that stress may relate to reported SSC increases because of $u^{+}$is discussed.
\end{abstract}

In tomato fruit, the wild-type green shoulder is characterized by an area of dark green pigmentation on the proximal or stem end (shoulder) of unripe fruit because of increased chlorophyll concentration, the biosynthesis of which is activated by light (Smillie et al., 1999). A mutant of the wild-type allele called uniform ripening ( $u$ gene) was first described by Yeager (1935). Bohn and Scott (1945) reported on a mutant called uniform gray-green ( $u g$ gene) that is identical to the $u$ phenotype, although Reynard (1952) thought he could distinguish the two genes. The phenotype of these two genes has uniform light green pigmentation on unripe fruit from the stem end to the distal or blossom end and will be referred to as uniform green hereafter. In the last four decades, tomato breeders have generally selected for uniform green fruit because of observations that green shoulders were associated with increased incidence of YS in ripening fruit (Picha, 1987; Picha and Hall, 1981; Scott, 2014; Venter, 1965; Yeager, 1935). YS is a ripening disorder in which all or part of the shoulder pericarp does not fully ripen, resulting in a hard yellow or green section distinctly demarked from the normally ripened tissue (Picha, 1987; Scott, 2014). YS has been classified as a form of blotchy ripening that occurs on the shoulder of the tomato (Picha and Hall, 1981) because both involve sections of fruit that remain yellow when the rest of the fruit turns red. However, blotchy ripening and YS

Received for publication 8 June 2017. Accepted for publication 21 Aug. 2017. ${ }^{1}$ Corresponding author. E-mail: jwsc@ufl.edu. are usually quite distinct from each other, and some genotypes are susceptible to only one or the other. Francis et al. (2000) described a second disorder they refer to as YS disorder where, in processing genotypes, uniform green fruit appear normal red on the outside, but when the peel is removed, pericarp tissue in the shoulder is yellow. A third discoloration described by Francis et al. (2000) is a yellow ring around the stem scar. This yellow ring is generally associated with white tissue formation in the pericarp of the fruit interior and may also be an indicator of the subcutaneous YS described by Francis et al. (2000). However, in this article, it was not considered as a form of YS as it only relates to white tissue formation in Florida (J.W. Scott, personal observations). Fruit with YS have no market value and are culled during packing (Picha, 1987; Venter, 1965). Besides genotypic effects, several other factors can lead to increased YS incidence. Low potassium increased the incidence and severity of YS (Venter, 1965). High temperatures, relative humidity, and light levels significantly increased the incidence of YS, with the effect of temperature dependent on light (Romero-Aranda et al., 2004). YS can be prevented by planting tolerant cultivars when harvesting ripening fruit in the sun. Otherwise, if fruit are harvested at the mature green stage and ripened in the dark with exogenous ethylene, they will not express YS (Scott, 2014).

Recently, Powell et al. (2012) reported that ripe fruit of 'Ailsa Craig', a cultivar with green-shouldered fruit, had significantly higher SSC than did 'Craigella', an isogenic 
Table 1. Parents, their immature fruit color genotypes, and phenotypic descriptions.

\begin{tabular}{lll}
\hline Parental lines & \multicolumn{1}{c}{ Gene-gene name } & \multicolumn{1}{c}{ Immature fruit color description } \\
\hline LA 3035 & $u$-uniform ripening & Fruit are uniformly light green from top to bottom. \\
NC 84173 & $u$-uniform ripening & Same as above \\
LA 0021 & $u g$-uniform gray-green & Same appearance as uniform ripening. \\
'Walter' & $u^{+}$-green shoulder (wild type) & Fruit shoulders are dark green when exposed to (sun) light. Base of fruit is light green. \\
'Micro-Tom' & $u^{\mathrm{Pg}-\text { pale green }}$ & Pale green; fruit are uniformly lighter green than $u$ and $u g$, they have a near whitish appearance \\
Fla. 7956 & $u^{\mathrm{Ag}}$ - apple green & Fruit are uniformly dark green from top to bottom, darker than $u$ and $u g$ but not as dark as \\
& & high pigment $(h p-1$ or $h p-2$ genes) or dark green $(d g$ gene). Foliage is also dark green. \\
Fla. 8033 & ?- medium-green shoulder & Fruit shoulder is slightly darker than that of $u$ and $u g$ but not as dark as green shoulder. \\
\hline
\end{tabular}

cultivar with uniform green $(u)$ shoulders. These authors concluded that tomato breeders, in selecting for the $u$ gene, had inadvertently selected for lower SSC and thus reduced fruit quality in both fresh and processed tomato cultivars. In another study, Nguyen et al. (2014) found a similar trend in that SSC was from $20 \%$ to $40 \%$ higher in $u^{+}$types than in $u$ types of tomatoes. The findings of Powell et al. (2012) and Nguyen et al. (2014) were surprising because it was widely accepted that fruit were sinks for photosynthetic activity with most photo-assimilates translocated from leaves, with tomato fruit themselves being only small contributors (Hackel et al., 2006; Zanor et al., 2009).

In addition to $u$ and $u g$, there are other green tomato fruit phenotypes that have been reported and/or observed in tomato breeding programs. Reynard (1956) reported that dark-pigmented fruit and/or foliage types were found in a rogue plant of an unknown line at a Campbell's farm (Riverton, NJ) and called this 'Webb's Special' ('Black Queen'). From this line, at least two genes, high pigment $1(\mathrm{hp}-1)$ and high pigment $2(h p-2)$, were discovered, and these had very dark green fruit and foliage (Reynard, 1960). Reynard (1960) also described fruit from 'Ontario' called apple, which hereafter will be called apple green, where the fruit are uniformly dark green with pigmentation lighter than that of high pigment but darker green than that of $u$ and $u g$. The foliage of apple green is also dark green, which may be a pleiotropic effect of a single gene. In the same report, Reynard (1960) briefly described fruit from 'Niihau' as being whitish green, and this phenotype may be similar to the fruit of 'Micro-Tom' that we refer to as pale green where the fruit are lighter than uniform green and are almost white in appearance. Another phenotype seen in the field but not reported we call medium-green shoulder, where fruit shoulders are darker green than those with $u$ or $u g$ but not as dark as fruit with green shoulders $\left(u^{+}\right)$. There has been little information published on the latter three phenotypes, but Mattia and Scott (2014) recently proposed the gene symbols for apple green and pale green to be $u^{\mathrm{Ag}}$ and $u^{\mathrm{Pg}}$, respectively, because they were both allelic at the $u$ locus.

There are no published data showing that green shoulders are associated with increased YS incidence, only observations. We do know that some green-shouldered cultivars have tolerance, and this has been reported for the 'Walter' tomato (Strobel et al., 1969). However, in general, it is believed that greenshouldered fruit absorb more light and subsequently obtain more heat than uniform green fruit, and this leads to enhanced YS expression (Scott, 2014). Perhaps, then, the darker $u^{\mathrm{Ag}}$ genotypes would also be prone to YS, and the very light $u^{\mathrm{Pg}}$ genotypes would be the least prone to YS. It is also important to know if there is a general trend for higher SSC to be associated with green shoulder vs. $u$ and/or other fruit color genotypes as this would suggest a breeding shift back to $u^{+}$to attain better fruit quality. Thus, the objectives of the present work were to determine the effects of $u^{+}, u, u g, u^{\mathrm{Ag}}, u^{\mathrm{Pg}}$, and medium-green shoulder on YS and SSC. A secondary objective was to evaluate the use of a colorimeter in distinguishing the immature fruit color phenotypes.

\section{Materials and Methods}

Plant materials and experimental design. Parental inbreds, their fruit genotypes, and descriptions are presented in Table 1. Those with an "Fla." designation were from the tomato breeding program at the University of Florida, Gulf Coast Research and Education Center (Wimauma, FL), one line with an "NC" designation was from the tomato breeding program at North Carolina State University, Mountain Crops 
Research and Extension Center (Mills River, NC), and those with an "LA" designation were from the Tomato Genetics Resource Center (Davis, CA). All parents with the exception of Fla. 8033 (medium-green shoulder) were crossed in all combinations without reciprocals in Fall 1999 to create $14 \mathrm{~F}_{1} \mathrm{~s}$. Subsequently, in Spring 2000, the $14 \mathrm{~F}_{1} \mathrm{~s}$ were allowed to self-pollinate to produce $\mathrm{F}_{2}$ seed. Fla. 8033 was crossed with each of the other parents in Fall 2012 to produce five $F_{1}$ s (the cross with green-shouldered 'Walter' failed), and $F_{2}$ seed was obtained in Spring 2013. In Spring 2013, additional crosses were made between parental lines to generate more $F_{1}$ seed for the subsequent season. For Fall 2013, seed of the two isogenic inbreds differing in shoulder color, 'Ailsa Craig' $\left(u^{+}\right)$and 'Craigella' $(u)$, were obtained from the Tomato Genetics Resource Center.

Field EXPERIMENT SPRING 2013. Seed were sown on $21 \mathrm{Feb}$. 2013 for parents, $F_{1} s$, and $F_{2} s$ in a greenhouse. On 24 Mar., seedlings were transplanted to the field on $20-\mathrm{cm}-\mathrm{high}, 81-\mathrm{cm}$ wide raised beds of EauGallie fine sand covered with plastic mulch. All genotypes were planted in a randomized completeblock design with three blocks. Parents and $\mathrm{F}_{1} \mathrm{~s}$ had five plants per plot, and $\mathrm{F}_{2} \mathrm{~s}$ had 15 plants per plot. There were no $\mathrm{F}_{2} \mathrm{~S}$ arising from Fla. 8033, the medium-green parent. Plants were spaced $46 \mathrm{~cm}$ apart within plots that were $91 \mathrm{~cm}$ apart in rows, with $152 \mathrm{~cm}$ between rows. Recommended fertilizer and insecticide programs were followed (Olson et al., 2011). Plants were grown with stake culture and drip irrigated.

Phenotyping immature fruit color. Sun-exposed green fruit were visually phenotyped for each plant from 22 to 24 June using parental fruit phenotypes as standards. The phenotypic groups were uniform (the $u$ and $u g$ genes were both in this class because of indistinguishable phenotypes), pale green, medium green, apple green, green shoulder, and green shoulder/apple green, where the latter was a combination of parental phenotypes and was scored accordingly. From 25 June to 1 July, objective color measurements were measured with a colorimeter (Chroma Meter CR-400/410; Konica-Minolta, Tokyo, Japan). Two green fruit per plant were harvested: one sun-exposed fruit and one fruit shaded by the plant canopy. The sun-exposed fruit were harvested to help validate the visual phenotype ratings, whereas canopy-covered fruit were harvested to show any differences in color expression due to differences in light exposure. Two measurements per fruit were taken: one on the shoulder and one on the blossom end (base). Measurements were expressed as $L^{*} a^{*} b^{*}$ (CIELAB) color space values. It was found that $\mathrm{L}^{*}$ scores indicating lightness provided the best differentials between the phenotypes studied. Analysis of variance (ANOVA) on fruit was done using the general linear model, and estimates of means and variances were made using SAS (version 9.2; SAS Institute, Cary, NC). Means were separated using Duncan's multiple range tests on $\mathrm{L}^{*}$ scores.

Soluble solids. On 27 June, two table-ripe fruit per plant were harvested, and soluble solids were measured for each using a digital refractometer (Palm Abbe model PA201; MISCO, Cleveland, $\mathrm{OH}$ ), and the two scores were averaged. The averaged SSC measurements were matched with visual phenotypic color data on a per plant basis. An ANOVA on SSC measurements was done using the general linear model procedure, and Duncan's multiple range tests were used to determine differences in the means. Estimates of means and variances were calculated with SAS (version 9.2) using the Proc Means procedure.
YelLow ShOulder. For this analysis, two plants per plot were randomly chosen from the parents and $F_{1}$ s, whereas $50 F_{2}$ plants per block (150 total) representing the segregating phenotypes in nearly equal numbers were randomly selected. For each plant, all red ripe fruit were harvested and divided into groups with and without YS. Counts of each group were made, and the percentage of YS was calculated as the number of fruit with YS divided by the total number of fruit. For analysis, data were transformed with the arcsine transformation (angular transformation), but the percentages are presented for clarity. An ANOVA on YS scores was performed using the general linear model procedure in SAS (version 9.2). Mean differences were determined using Duncan's multiple range test and variances were calculated with SAS using the Proc Means procedure (version 9.2).

Field EXPERIMENT fall 2013. The same genotypes as in Spring 2013 were used along with seed of 'Ailsa Craig' and 'Craigella'. These were planted in five plant plots per block. On 7 Aug. 2013, seed were sown for parents, $F_{1} s$, and $F_{2} s$. Sun-exposed green fruit were phenotyped from 25 to 30 Nov.,

Table 2. Tomato fruit shoulder and base (blossom end) CIE L* score means and standard errors for parents and $F_{1} s$ grouped by phenotype for Spring and Fall 2013 field seasons.

\begin{tabular}{lllccc}
\hline & & \multicolumn{3}{c}{ Darkness $\left(\mathrm{L}^{*}\right)^{\mathrm{z}}$} \\
\cline { 3 - 6 } & & \multicolumn{3}{c}{ Shoulder } & \multicolumn{2}{c}{ Base } \\
\hline \multirow{4}{*}{ Season } & Phenotype & Mean & SE & Mean & SE \\
& Apple green & 52.26 & 0.498 & 55.52 & 0.605 \\
& Pale green & 68.14 & 0.811 & 66.62 & 0.767 \\
& Medium green & 58.01 & 0.794 & 61.72 & 0.948 \\
& Green shoulder & 54.36 & 0.559 & 62.32 & 0.518 \\
& Uniform green & 58.13 & 0.466 & 62.07 & 0.465 \\
& Green shoulder/apple green & 52.26 & 1.066 & 55.45 & 1.118 \\
& Apple green & 50.28 & 0.651 & 52.92 & 0.779 \\
& Pale green & 67.79 & 1.230 & 64.48 & 1.280 \\
& Medium green & 52.77 & 1.220 & 56.68 & 1.170 \\
& Green shoulder & 47.44 & 0.529 & 57.61 & 0.509 \\
& Uniform green & 56.91 & 0.519 & 56.27 & 0.532 \\
& Green shoulder/apple green & 44.17 & 1.206 & 51.53 & 0.984 \\
\hline
\end{tabular}

$\overline{{ }^{2} \text { Measure of lightness and darkness determined with a colorimeter }}$ where higher scores indicate more lightness and lower scores more darkness.

Table 3. Tomato fruit shoulder and base (blossom end) CIE $L^{*}$ scores for sun-exposed fruit of $F_{2}$ plants grouped by phenotype over Fall and Spring 2013 field seasons.

\begin{tabular}{lcccc}
\hline & \multicolumn{4}{c}{ Darkness $\left(\mathrm{L}^{*}\right)^{\mathrm{z}}$} \\
\cline { 2 - 5 } & \multicolumn{2}{c}{ Shoulder } & \multicolumn{2}{c}{ Base } \\
\hline Phenotype & Spring & Fall & Spring & Fall \\
Pale green & $65.14 \mathrm{a}^{\mathrm{y}}$ & $63.28 \mathrm{a}$ & $66.42 \mathrm{a}$ & $64.48 \mathrm{a}$ \\
Uniform green & $59.11 \mathrm{~b}$ & $55.57 \mathrm{~b}$ & $61.77 \mathrm{~b}$ & $56.27 \mathrm{~b}$ \\
Medium green & $-{ }^{\mathrm{x}}$ & $53.06 \mathrm{c}$ & $-{ }^{\mathrm{x}}$ & $56.68 \mathrm{~b}$ \\
Green shoulder & $56.91 \mathrm{c}$ & $49.20 \mathrm{e}$ & $61.92 \mathrm{~b}$ & $57.61 \mathrm{~b}$ \\
Apple green & $55.16 \mathrm{~d}$ & $51.56 \mathrm{~d}$ & $55.98 \mathrm{c}$ & $52.92 \mathrm{c}$ \\
Green shoulder/apple green & $52.12 \mathrm{e}$ & $45.90 \mathrm{f}$ & $57.17 \mathrm{c}$ & $51.53 \mathrm{~d}$ \\
\hline
\end{tabular}

$\overline{{ }^{z} \text { Measure of lightness and darkness determined with a colorimeter }}$ where higher scores indicate more lightness and lower scores more darkness.

${ }^{\mathrm{y}}$ Mean separation in columns by Duncan's multiple range test at $P \leq$ 0.05 .

${ }^{\mathrm{x}}$ No $\mathrm{F}_{2}$ plants available. 
and color was measured from 1 to 8 Dec. SSC measurements were taken from 9 to 17 Dec. and on 20 Dec.; 152 plants were harvested and graded for YS (see the Results section). All other procedures were the same as those used in Spring 2013.

Table 4. Mean CIE L* scores for shoulders and bases (blossom ends) of immature green tomato fruit that were canopy covered and sun exposed from $\mathrm{F}_{2}$ plants grouped by phenotype in Spring and Fall 2013 seasons.

\begin{tabular}{|c|c|c|c|c|}
\hline \multirow{2}{*}{ Season } & \multirow[b]{2}{*}{ Fruit location } & \multirow[b]{2}{*}{ Phenotype } & \multicolumn{2}{|c|}{ Darkness $\left(\mathrm{L}^{*}\right)^{\mathrm{z}}$} \\
\hline & & & Canopy & Sun \\
\hline \multirow[t]{10}{*}{ Spring 2013} & Shoulder & Apple green & 55.27 & 55.16 \\
\hline & & Pale green & 66.70 & 65.14 \\
\hline & & Green shoulder & $59.29^{*}$ & 56.91 \\
\hline & & Uniform green & $61.18^{*}$ & 59.11 \\
\hline & & Green shoulder apple base & 53.92 & 52.12 \\
\hline & Base & Apple green & 56.16 & 55.98 \\
\hline & & Pale green & 67.11 & 66.42 \\
\hline & & Green shoulder & 62.42 & 61.92 \\
\hline & & Uniform green & 62.44 & 61.77 \\
\hline & & Green shoulder apple base & $57.30^{*}$ & 57.17 \\
\hline \multirow[t]{12}{*}{ Fall 2013} & Shoulder & Apple green & $53.58^{*}$ & 51.56 \\
\hline & & Pale green & 64.21 & 63.28 \\
\hline & & Medium green & $55.89^{*}$ & 53.06 \\
\hline & & Green shoulder & $54.20^{*}$ & 49.20 \\
\hline & & Uniform green & $57.06^{*}$ & 55.57 \\
\hline & & Green shoulder apple base & $51.59^{*}$ & 45.90 \\
\hline & Base & Apple green & $53.78^{*}$ & 52.92 \\
\hline & & Pale green & 65.03 & 64.48 \\
\hline & & Medium green & 56.92 & 56.68 \\
\hline & & Green shoulder & 57.71 & 57.61 \\
\hline & & Uniform green & $57.00 *$ & 56.27 \\
\hline & & Green shoulder apple base & 52.43 & 51.53 \\
\hline
\end{tabular}

${ }_{\mathrm{z}}$ Measure of lightness and darkness determined with a colorimeter where higher scores indicate more lightness and lower scores more darkness.

*Indicates significance between canopy and sun at $P \leq 0.05$.

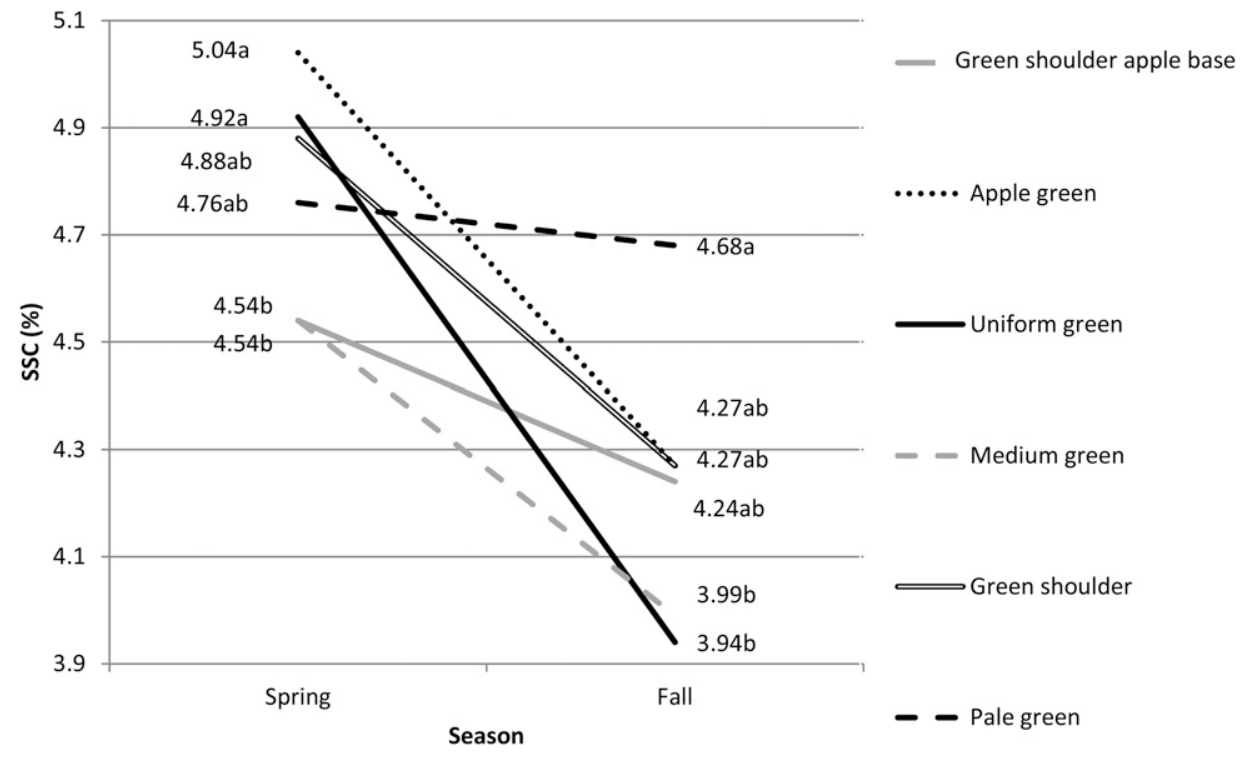

Fig. 2. Soluble solids concentration (SSC) for tomato parents and $\mathrm{F}_{1}$ s grouped by phenotype over Spring and Fall 2013 seasons. Mean separation within seasons by Duncan's multiple range test at $P \leq 0.05$.

\section{Results}

GreEN FRUIT COLOR. All color analyses were based on CIE $\mathrm{L}^{*}$, a measure of lightness, because this variable best related to phenotypic separation. ANOVA indicated that there was an interaction of season and immature fruit color phenotype among parents and $F_{1} s$ (data not shown). Thus, data are presented separately for each season. The uniform fruit color phenotypes pale green, uniform green, and apple green were fairly constant over seasons, whereas green shoulder/apple green, medium green, and green shoulder were lighter in the spring than in the fall (Fig. 1). There was sometimes difficulty in distinguishing differences in immature green color phenotypes, and there was an overlap of standard errors for some of the phenotypes (Table 2).

Parents and $\mathrm{F}_{1} \mathrm{~s}$ were grouped by phenotype as were 19 families of $F_{2} s$. For shoulder color, the significant differences were the same for both groups (Tables 2 and 3). In the spring, the order of shoulder color from darkest to lightest was as follows: green shoulder/apple green $>$ apple green $>$ green shoulder $>$ uniform green and medium-green shoulder $>$ pale green. In the fall, the darkest to lightest order was green shoulder/apple green $>$ green shoulder $>$ apple green $>$ medium-green shoulder $>$ uniform green $>$ pale green. Although there were many significant differences in the means, there was sometimes overlap in colorimeter readings between phenotypic categories, so the colorimeter could not be used to unequivocally separate all of the phenotypes that were based on an overall observation of many fruit. For fruit base color, there were three significant groups in order from darkest to lightest: apple green with and without green shoulder $>$ green shoulder, medium-green shoulder, and uniform green $>$ pale green.

Shoulder color of sun-exposed fruit was significantly darker than canopy-covered fruit over both seasons for the phenotypes apple green, medium green shoulder, green shoulder, and uniform green (Table 4). The green shoulder/apple green sunexposed fruit were significantly darker than canopy-covered fruit only in the fall. There were fewer significant differences in fruit base color between sun-exposed vs. canopy-covered fruit (Table 4). In the spring, sun-exposed fruit base colors were significantly darker than canopy-covered fruit base colors for medium-green shoulder and greenshoulder/apple green phenotypes only. In the fall, only apple green and uniform green phenotypes had significant differences.

SSC. Parents and $\mathrm{F}_{1} \mathrm{~s}$ grown over both seasons were grouped by phenotype, and ANOVA indicated that season, phenotype, and the interaction of season $\times$ phenotype were all significant (data not shown). The overall mean for parents and $F_{1} \mathrm{~S}$ was significantly higher in the spring (4.67) than in the fall (4.23) $(F \leq 0.05)$. The interaction occurred because the SSC for pale green phenotypes did not drop much from spring to fall, whereas there were significant decreases for the 
other phenotypes (Fig. 2). In Spring 2013, apple green and uniform green phenotypes had significantly higher SSC than the green shoulder/ apple green and medium-green phenotypes, whereas other phenotypes were intermediate and not significantly different from either of these groups (Fig. 2). In Fall 2013, pale green had significantly greater SSC than uniform green and mediumgreen phenotypes, whereas other phenotypes were intermediate and not significantly different from either of these groups (Fig. 2). Thus, for parents and $\mathrm{F}_{1} \mathrm{~s}$ grouped by phenotype, there was no consistent relationship of phenotype with SSC over both seasons.

Analysis of individual $\mathrm{F}_{2}$ families for each season or when averaged over both seasons revealed no pattern where SSC corresponded well with any particular phenotype (Table 5). There was no trend where there was a significant difference in one season that repeated itself in the other season. In fact, when data were averaged over both seasons, there was no significant difference in SSC between phenotypic groups within any of the $F_{2}$ families. When $\mathrm{F}_{2}$ data were grouped over all families by fruit phenotype for all those grown in both seasons, the SSC in the spring season was higher than that in the fall (Table 6). In the fall, green shoulder/apple green and apple green plants had fruit with higher SSC than all other phenotypes except for medium green/ apple green. Medium green/apple green had significantly higher SSC than uniform green, but there were no other differences among the phenotypes (Table 6). Thus, there was no consistent association of SSC with fruit color phenotype across the two seasons. In the fall, both phenotypes with the highest SSC were apple green with or without green shoulders. There was no evidence that green-shoulder phenotypes in the $\mathrm{F}_{2}$ had increased SSC (Table 6). In addition, the SSC of 'Ailsa Craig' $\left(u^{+}\right)$and 'Craigella' $(u)$ fruit did not significantly differ when tested in Fall 2013 (Fig. 3).

Yellow SHOULDER. ANOVA indicated that season and phenotype were both significant for YS incidence, but there was no significant
Table 5. Tomato soluble solids content for 19 families of parents, $F_{1} s$, and phenotypic groups of plants that segregated in the $F_{2}$ over Spring and Fall 2013 seasons.

\begin{tabular}{|c|c|c|c|c|c|}
\hline \multirow[b]{2}{*}{ Family } & \multirow[b]{2}{*}{ Generation } & \multirow[b]{2}{*}{ Genotype/phenotype ${ }^{z}$} & \multicolumn{3}{|c|}{ Soluble solids concn $(\%)$} \\
\hline & & & Spring 2013 & Fall 2013 & Avg \\
\hline \multirow[t]{5}{*}{1} & $\mathrm{P}_{1}(\mathrm{LA} 3035)$ & $u$ & $4.63 \mathrm{~b}^{\mathrm{y}}$ & $4.12 \mathrm{ab}$ & $4.38 \mathrm{~b}$ \\
\hline & $\mathrm{P}_{2}(\mathrm{LA} 0021)$ & $u g$ & $5.39 \mathrm{a}$ & $4.29 \mathrm{a}$ & $4.84 \mathrm{a}$ \\
\hline & $\mathrm{F}_{1}$ & $u^{+}$ & $4.61 \mathrm{~b}$ & $3.81 \mathrm{ab}$ & $4.21 \mathrm{~b}$ \\
\hline & $\mathrm{F}_{2}$ & $u / u g$ & $5.10 \mathrm{a}$ & $3.71 \mathrm{~b}$ & $4.41 \mathrm{~b}$ \\
\hline & $\mathrm{F}_{2}$ & $u^{+}$ & $5.31 \mathrm{a}$ & $3.81 \mathrm{ab}$ & $4.56 \mathrm{~b}$ \\
\hline \multirow[t]{6}{*}{2} & $\mathrm{P}_{1}(\mathrm{LA} 3035)$ & $u$ & $4.63 \mathrm{a}$ & $4.12 \mathrm{~b}$ & $4.38 \mathrm{a}$ \\
\hline & $\mathrm{P}_{2}$ ('Walter') & $u^{+}$ & $3.82 \mathrm{a}$ & $3.85 \mathrm{~b}$ & $3.84 \mathrm{a}$ \\
\hline & $\mathrm{F}_{1}$ & $u^{+}$ & $4.88 \mathrm{a}$ & $4.77 \mathrm{a}$ & $4.83 \mathrm{a}$ \\
\hline & $\mathrm{F}_{2}$ & $u$ & $5.09 \mathrm{a}$ & $4.21 \mathrm{~b}$ & $4.65 \mathrm{a}$ \\
\hline & $\mathrm{F}_{2}$ & $u^{+}$ & $5.56 \mathrm{a}$ & $4.11 \mathrm{~b}$ & $4.84 \mathrm{a}$ \\
\hline & $\mathrm{P}_{1}(\mathrm{LA} 0021)$ & $u g$ & $5.39 \mathrm{a}$ & $4.23 \mathrm{a}$ & $4.81 \mathrm{a}$ \\
\hline \multirow[t]{5}{*}{3} & $\mathrm{P}_{2}$ ('Walter') & $u^{+}$ & $3.82 \mathrm{~b}$ & $3.85 \mathrm{ab}$ & $3.84 \mathrm{~b}$ \\
\hline & $\mathrm{F}_{1}$ & $u^{+}$ & $5.24 \mathrm{a}$ & $4.34 \mathrm{a}$ & $4.79 \mathrm{a}$ \\
\hline & $\mathrm{F}_{2}$ & $u^{+}$ & $4.95 \mathrm{a}$ & $4.04 \mathrm{ab}$ & $4.50 \mathrm{a}$ \\
\hline & $\mathrm{F}_{2}$ & $u / u g$ & $5.30 \mathrm{a}$ & $3.68 \mathrm{~b}$ & $4.49 \mathrm{a}$ \\
\hline & $\mathrm{P}_{1}(\mathrm{LA} 0021)$ & $u g$ & $5.39 \mathrm{~b}$ & $4.23 \mathrm{a}$ & $4.81 \mathrm{~b}$ \\
\hline \multirow[t]{5}{*}{4} & $\mathrm{P}_{2}(\mathrm{NC} 84173)$ & $u$ & $5.51 \mathrm{~b}$ & $4.01 \mathrm{a}$ & $4.76 \mathrm{~b}$ \\
\hline & $\mathrm{F}_{1}$ & $u^{+}$ & $6.09 \mathrm{a}$ & $\underbrace{\mathrm{y}}$ & - \\
\hline & $\mathrm{F}_{2}$ & $u / u g$ & $4.89 \mathrm{~b}$ & $4.01 \mathrm{a}$ & $4.45 \mathrm{~b}$ \\
\hline & $\mathrm{F}_{2}$ & $u^{+}$ & $5.48 \mathrm{~b}$ & $3.89 \mathrm{a}$ & $4.69 \mathrm{~b}$ \\
\hline & $\mathrm{P}_{1}(\mathrm{LA} 0021)$ & $u g$ & $5.39 \mathrm{a}$ & $4.22 \mathrm{bc}$ & $4.81 \mathrm{a}$ \\
\hline \multirow[t]{5}{*}{5} & $\mathrm{P}_{2}$ ('Micro-Tom') & $u^{\mathrm{Pg}}$ & $5.23 \mathrm{ab}$ & $4.50 \mathrm{~b}$ & $4.86 \mathrm{a}$ \\
\hline & $\mathrm{F}_{1}$ & $u^{+}$ & $4.53 \mathrm{~b}$ & $5.95 \mathrm{a}$ & $5.24 \mathrm{a}$ \\
\hline & $\mathrm{F}_{2}$ & $u^{+}$ & $4.75 \mathrm{ab}$ & $3.14 \mathrm{c}$ & $3.95 \mathrm{~b}$ \\
\hline & $\mathrm{F}_{2}$ & $u^{\mathrm{Pg}}$ & $5.21 \mathrm{ab}$ & $3.57 \mathrm{bc}$ & $4.39 \mathrm{ab}$ \\
\hline & $\mathrm{P}_{1}$ ('Walter') & $u^{+}$ & $3.82 \mathrm{c}$ & $3.88 \mathrm{ab}$ & $3.85 \mathrm{~b}$ \\
\hline \multirow[t]{5}{*}{6} & $\mathrm{P}_{2}(\mathrm{NC} 84173)$ & $u$ & $5.51 \mathrm{a}$ & $4.01 \mathrm{ab}$ & $4.76 \mathrm{a}$ \\
\hline & $\mathrm{F}_{1}$ & $u^{+}$ & $4.15 \mathrm{bc}$ & - & - \\
\hline & $\mathrm{F}_{2}$ & $u^{+}$ & $4.45 \mathrm{~b}$ & $3.75 \mathrm{~b}$ & $4.10 \mathrm{~b}$ \\
\hline & $\mathrm{F}_{2}$ & $u$ & $4.05 \mathrm{bc}$ & $4.35 \mathrm{a}$ & $4.20 \mathrm{~b}$ \\
\hline & $\mathrm{P}_{1}$ ('Walter') & $u^{+}$ & $3.82 \mathrm{~b}$ & $3.85 \mathrm{ab}$ & $3.84 \mathrm{ab}$ \\
\hline \multirow[t]{6}{*}{7} & $\mathrm{P}_{2}$ ('Micro-Tom') & $u^{\mathrm{Pg}}$ & $5.23 \mathrm{a}$ & $4.61 \mathrm{a}$ & $4.92 \mathrm{a}$ \\
\hline & $\mathrm{F}_{1}$ & $u^{+}$ & - & $3.70 \mathrm{ab}$ & - \\
\hline & $\mathrm{F}_{2}$ & $u^{+}$ & $3.76 \mathrm{~b}$ & $3.40 \mathrm{ab}$ & $3.58 \mathrm{~b}$ \\
\hline & $\mathrm{F}_{2}$ & $u^{\mathrm{Pg}}$ & $4.11 \mathrm{~b}$ & $3.07 \mathrm{~b}$ & $3.59 \mathrm{~b}$ \\
\hline & $\mathrm{F}_{2}$ & $u^{+} / u^{\mathrm{Ag}}$ & $4.94 \mathrm{ab}$ & $4.45 \mathrm{a}$ & $4.70 \mathrm{ab}$ \\
\hline & $\mathrm{P}_{1}$ ('Walter') & $u^{+}$ & $3.82 \mathrm{c}$ & $3.85 \mathrm{ab}$ & $3.84 \mathrm{c}$ \\
\hline \multirow[t]{6}{*}{8} & $\mathrm{P}_{2}$ (Fla. 7956) & $u^{\mathrm{Ag}}$ & $4.89 \mathrm{ab}$ & $4.39 \mathrm{a}$ & $4.64 \mathrm{a}$ \\
\hline & $\mathrm{F}_{1}$ & $u^{+} / u^{\mathrm{Ag}}$ & $5.11 \mathrm{a}$ & $4.13 \mathrm{ab}$ & $4.62 \mathrm{a}$ \\
\hline & $\mathrm{F}_{2}$ & $u^{\mathrm{Ag}}$ & $4.67 \mathrm{ab}$ & $3.74 \mathrm{~b}$ & $4.21 \mathrm{bc}$ \\
\hline & $\mathrm{F}_{2}$ & $u$ & $4.40 \mathrm{bc}$ & $4.27 \mathrm{ab}$ & $4.34 \mathrm{ab}$ \\
\hline & $\mathrm{F}_{2}$ & $u^{+}$ & $4.87 \mathrm{ab}$ & $4.39 \mathrm{a}$ & $4.63 \mathrm{a}$ \\
\hline & $\mathrm{P}_{1}(\mathrm{LA} 3035)$ & $u$ & $4.63 \mathrm{~b}$ & $4.11 \mathrm{ab}$ & $4.37 \mathrm{~b}$ \\
\hline \multirow[t]{5}{*}{9} & $\mathrm{P}_{2}$ (Fla. 7956) & $u^{\mathrm{Ag}}$ & $4.89 \mathrm{ab}$ & $4.38 \mathrm{a}$ & $4.64 \mathrm{ab}$ \\
\hline & $\mathrm{F}_{1}$ & $u^{\mathrm{Ag}}$ & $5.02 \mathrm{ab}$ & $4.29 \mathrm{ab}$ & $4.66 \mathrm{ab}$ \\
\hline & $\mathrm{F}_{2}$ & $u$ & $4.91 \mathrm{ab}$ & $3.85 \mathrm{~b}$ & $4.38 \mathrm{~b}$ \\
\hline & $\mathrm{F}_{2}$ & $u^{\mathrm{Ag}}$ & $5.12 \mathrm{a}$ & $4.50 \mathrm{a}$ & $4.81 \mathrm{a}$ \\
\hline & $\mathrm{P}_{1}(\mathrm{LA} 0021)$ & $u g$ & $5.39 \mathrm{a}$ & $4.22 \mathrm{ab}$ & $4.81 \mathrm{a}$ \\
\hline \multirow[t]{6}{*}{10} & $\mathrm{P}_{2}$ (Fla. 7956) & $u^{\mathrm{Ag}}$ & $4.89 \mathrm{ab}$ & $4.39 \mathrm{ab}$ & $4.64 \mathrm{a}$ \\
\hline & $\mathrm{F}_{1}$ & $u^{\mathrm{Ag}}$ & $4.70 \mathrm{~b}$ & $4.04 \mathrm{a}-\mathrm{c}$ & $4.37 \mathrm{a}$ \\
\hline & $\mathrm{F}_{2}$ & $u g$ & $4.94 \mathrm{~b}$ & $3.93 \mathrm{bc}$ & $4.21 \mathrm{a}$ \\
\hline & $\mathrm{F}_{2}$ & $u^{+} / u^{\mathrm{Ag}}$ & $5.18 \mathrm{ab}$ & $3.72 \mathrm{c}$ & $4.45 \mathrm{a}$ \\
\hline & $\mathrm{F}_{2}$ & $u^{\mathrm{Ag}}$ & $4.54 \mathrm{~b}$ & $4.59 \mathrm{a}$ & $4.56 \mathrm{a}$ \\
\hline & $\mathrm{P}_{1}$ (Fla. 7956) & $u^{\mathrm{Ag}}$ & $4.89 \mathrm{a}$ & $4.39 \mathrm{a}$ & $4.64 \mathrm{bc}$ \\
\hline
\end{tabular}

Continued next page 
Table 5. Continued.

\begin{tabular}{|c|c|c|c|c|c|}
\hline \multirow[b]{2}{*}{ Family } & \multirow[b]{2}{*}{ Generation } & \multirow[b]{2}{*}{ Genotype/phenotype $\mathrm{e}^{\mathrm{z}}$} & \multicolumn{3}{|c|}{ Soluble solids concn $(\%)$} \\
\hline & & & Spring 2013 & Fall 2013 & Avg \\
\hline \multirow[t]{5}{*}{11} & $\mathrm{P}_{2}(\mathrm{NC} 84173)$ & $u$ & $5.51 \mathrm{a}$ & $4.02 \mathrm{a}$ & $4.77 \mathrm{bc}$ \\
\hline & $\mathrm{F}_{1}$ & $u^{\mathrm{Ag}}$ & $5.60 \mathrm{a}$ & - & - \\
\hline & $\mathrm{F}_{2}$ & $u$ & $5.35 \mathrm{a}$ & $4.11 \mathrm{a}$ & $4.73 \mathrm{c}$ \\
\hline & $\mathrm{F}_{2}$ & $u^{\mathrm{Ag}}$ & $5.06 \mathrm{a}$ & $4.55 \mathrm{a}$ & $4.80 \mathrm{~b}$ \\
\hline & $\mathrm{P}_{1}$ (Fla. 7956) & $u^{\mathrm{Ag}}$ & $4.89 \mathrm{ab}$ & $4.39 \mathrm{a}$ & $4.64 \mathrm{ab}$ \\
\hline \multirow[t]{6}{*}{12} & $\mathrm{P}_{2}$ ('Micro-Tom') & $u^{\mathrm{Pg}}$ & $5.23 \mathrm{a}$ & $4.61 \mathrm{a}$ & $4.92 \mathrm{a}$ \\
\hline & $\mathrm{F}_{1}$ & $u$ & $4.69 \mathrm{ab}$ & $3.63 \mathrm{a}$ & $4.16 \mathrm{ab}$ \\
\hline & $\mathrm{F}_{2}$ & $u^{\mathrm{Pg}}$ & $2.70 \mathrm{c}$ & $3.54 \mathrm{a}$ & $3.12 \mathrm{~b}$ \\
\hline & $\mathrm{F}_{2}$ & $u^{\mathrm{Ag}}$ & $3.79 \mathrm{bc}$ & $3.55 \mathrm{a}$ & $3.66 \mathrm{ab}$ \\
\hline & $\mathrm{F}_{2}$ & $u$ & $4.65 \mathrm{ab}$ & $4.01 \mathrm{a}$ & $4.33 \mathrm{ab}$ \\
\hline & $P_{1}(\mathrm{LA} 3035)$ & $u$ & $4.63 \mathrm{ab}$ & $4.12 \mathrm{a}$ & $4.38 \mathrm{ab}$ \\
\hline \multirow[t]{5}{*}{13} & $\mathrm{P}_{2}$ ('Micro-Tom') & $u^{\mathrm{Pg}}$ & $5.23 \mathrm{a}$ & $4.60 \mathrm{a}$ & $4.92 \mathrm{a}$ \\
\hline & $\mathrm{F}_{1}$ & $u^{\mathrm{Pg}}$ & $4.18 \mathrm{~b}$ & $3.80 \mathrm{a}$ & $3.89 \mathrm{~b}$ \\
\hline & $\mathrm{F}_{2}$ & $u$ & $4.60 \mathrm{ab}$ & $3.11 \mathrm{a}$ & $3.86 \mathrm{~b}$ \\
\hline & $\mathrm{F}_{2}$ & $u^{\mathrm{Pg}}$ & $5.01 \mathrm{a}$ & $3.64 \mathrm{a}$ & $4.33 \mathrm{ab}$ \\
\hline & $\mathrm{P}_{1}$ (Fla. 8033) & $m g$ & - & $3.95 \mathrm{a}$ & NA \\
\hline \multirow[t]{6}{*}{14} & $\mathrm{P}_{2}(\mathrm{LA} 3035)$ & $u$ & - & $3.95 \mathrm{a}$ & NA \\
\hline & $\mathrm{F}_{1}$ & $m g$ & - & $3.73 \mathrm{a}$ & NA \\
\hline & $\mathrm{F}_{2}$ & $m g$ & - & $3.68 \mathrm{a}$ & NA \\
\hline & $\mathrm{F}_{2}$ & $u g$ & - & $4.71 \mathrm{a}$ & NA \\
\hline & $\mathrm{F}_{2}$ & $u$ & - & $3.81 \mathrm{a}$ & NA \\
\hline & $\mathrm{P}_{1}$ (Fla. 8033) & $m g$ & - & $3.95 \mathrm{bc}$ & NA \\
\hline \multirow[t]{4}{*}{15} & $\mathrm{P}_{2}(\mathrm{LA} 0021)$ & $u g$ & - & $4.23 \mathrm{ab}$ & NA \\
\hline & $\mathrm{F}_{1}$ & $m g$ & - & $3.93 \mathrm{bc}$ & NA \\
\hline & $\mathrm{F}_{2}$ & $m g$ & - & $3.66 \mathrm{c}$ & NA \\
\hline & $\mathrm{P}_{1}$ (Fla. 8033) & $m g$ & - & $3.95 \mathrm{a}$ & NA \\
\hline \multirow[t]{5}{*}{16} & $\mathrm{P}_{2}(\mathrm{NC} 84173)$ & $u$ & - & $4.02 \mathrm{a}$ & NA \\
\hline & $\mathrm{F}_{1}$ & $m g$ & - & $3.63 \mathrm{a}$ & NA \\
\hline & $\mathrm{F}_{2}$ & $u$ & - & $3.57 \mathrm{a}$ & NA \\
\hline & $\mathrm{F}_{2}$ & $m g$ & - & $3.61 \mathrm{a}$ & NA \\
\hline & $\mathrm{P}_{1}($ Fla. 8033) & $m g$ & - & $3.95 \mathrm{a}$ & NA \\
\hline \multirow[t]{5}{*}{17} & $\mathrm{P}_{2}$ ('Micro-Tom') & $u^{\mathrm{Pg}}$ & - & $4.68 \mathrm{a}$ & NA \\
\hline & $\mathrm{F}_{1}$ & $m g$ & - & $2.91 \mathrm{c}$ & NA \\
\hline & $\mathrm{F}_{2}$ & $m g$ & - & $3.01 \mathrm{bc}$ & NA \\
\hline & $\mathrm{F}_{2}$ & $u^{\mathrm{Ag}}$ & - & $3.86 \mathrm{ab}$ & NA \\
\hline & $\mathrm{P}_{1}$ (Fla. 8033) & $m g$ & - & $3.95 \mathrm{a}$ & NA \\
\hline \multirow[t]{7}{*}{18} & $\mathrm{P}_{2}$ (Fla. 7956) & $u^{\mathrm{Ag}}$ & - & $4.39 \mathrm{a}$ & NA \\
\hline & $\mathrm{F}_{1}$ & $u^{\mathrm{Ag}}$ & - & $3.25 \mathrm{~b}$ & NA \\
\hline & $\mathrm{F}_{2}$ & $m g$ & - & $4.11 \mathrm{a}$ & NA \\
\hline & $\mathrm{F}_{2}$ & $u$ & - & $4.11 \mathrm{a}$ & NA \\
\hline & $\mathrm{F}_{2}$ & $u^{\mathrm{Ag}}$ & - & $4.29 \mathrm{a}$ & NA \\
\hline & $\mathrm{F}_{2}$ & $m g / u^{\mathrm{Ag}}$ & - & $4.48 \mathrm{a}$ & NA \\
\hline & $\mathrm{F}_{2}$ & $m g / u^{\mathrm{Ag}}$ & - & $4.48 \mathrm{a}$ & NA \\
\hline
\end{tabular}

${ }^{\mathrm{z}}$ Phenotypes are described by gene symbols (genotype) that control them as follows: $u=$ uniform ripening; $u g=$ uniform gray-green; $u / u g=$ uniform color can be due to either gene as they are indistinguishable; $\mathrm{mg}=$ medium-green shoulder; $u^{\mathrm{Pg}}=$ pale green; $u^{\mathrm{Ag}}=$ apple green; $u^{+}=$green shoulder; $u^{+} / u^{\mathrm{Ag}}=$ green shoulder apple base; $m g / u^{\mathrm{Ag}}=$ medium-green shoulder apple base.

${ }^{\mathrm{y}}$ Mean separation in columns within families by Duncan's multiple range test at $P \leq 0.05$.

${ }^{x}$ Represents no measureable fruit. Families 14 through 18 had no individuals planted in the Spring 2013 season.

$\mathrm{NA}=$ not applicable.

interaction between phenotypes and seasons (data not shown). The average YS incidence for parents and $\mathrm{F}_{1} \mathrm{~s}$ in the spring was $17.6 \%$, and this was significantly greater than that the fall where there was $13.4 \%$ YS $(P \leq 0.05)$. There was considerable variation for $Y S$ among the parents and $F_{1} s$, limiting significant differences between genotypes, yet some trends can be seen
(Table 7). There was a tendency for genotypes with green or medium shoulders to have higher percentages of YS, but again differences were not always significant with other genotypes. The major source of green shoulder in these studies was 'Walter', but it tended to have a lower percentage of YS. Fla. 8033, the parent with medium-green shoulders, was susceptible as it had the highest percentage of YS. Fla. 7956, the apple green parent, had no YS fruit. Three hybrids with Fla. 7956 also had no YS including the Fla. $8033 \times$ Fla. 7956 hybrid. Although Fla. 8033 had the highest percentage of YS, it had mediumgreen shoulder, whereas the aforementioned hybrid fruit were apple green, and this likely contributed to the YS response. The effects of shoulder color on YS were more clearly seen in the $F_{2}$ generation where segregating plants were grouped by phenotype (Table 8 ). In the spring, phenotypes with green-shouldered fruit had a higher incidence of YS than all other phenotypes except green shoulder/ apple green which did not differ from any other phenotype (Table $8)$. In the fall, plants with mediumgreen shoulders had significantly greater YS incidence than all other phenotypes except for those with green shoulders, which were not significantly different from the rest of the phenotypes (Table 8). 'Ailsa Craig', a cultivar with green-shouldered fruit, had significantly higher YS incidence than its isogenic uniform green fruit counterpart 'Craigella' (Fig. 3).

\section{Discussion}

Phenotyping immature fruit COLOR GENOTYPES. Phenotyping was done by observing many of the fruit on each plant to account for differences in sun exposure. Our secondary objective was to evaluate the ability of a colorimeter to distinguish phenotypes. The $\mathrm{L}^{*}$ score from the colorimeter helped to confirm the phenotyping and quantify differences in the green color of the immature fruit color genotypes studied, but separation of some of the genotypes was not possible (Tables 2-4). Present attempts at differentiating between $u$ and $u g$ types using a colorimeter were not successful; therefore, data for both phenotypes were combined under the title "uniform green." 
This was contrary to Kemp and Nonnecke (1960) who reported that they were able to differentiate between $u$ and $u g$ mutants using a colorimeter. Although means of many of the genotypes studied here could be separated with the colorimeter, sometimes individual fruit had $\mathrm{L}^{*}$ scores that overlapped with those of another genotype (data not shown). Shoulder color of sunexposed fruit was often darker than that of canopy-covered fruit, and this adds another factor to consider in the phenotyping process (Table 4). Immature green fruit phenotypes are activated by sunlight (Smillie et al., 1999), and fruit that are shaded by the canopy often do not have the same appearance as their sun-exposed counterparts. Pale green was not significantly different between sun-exposed and canopy-covered fruit; perhaps because there was not enough chlorophyll to be affected. Green shoulder/apple green types in the spring were also not significantly different between sun-exposed and canopy covered fruit. This may be due to higher solar radiation, causing lighter sun exposed fruit for this phenotype. Thus, there may be some ambiguity in phenotyping these genotypes both with and without a colorimeter.

To distinguish green shoulder and medium-green shoulder fruit, observation of sun-exposed fruit was required. Pale green and apple green were easily distinguished from each other, but separating either of these from uniform green was not always as easy. The dark green foliage of apple green was a helpful

Table 6. Mean tomato soluble solids concentration for $\mathrm{F}_{2} \mathrm{~s}$ grouped by phenotype for Spring and Fall 2013 seasons.

\begin{tabular}{lcc}
\hline & \multicolumn{2}{c}{ Soluble solids concn (\%) } \\
\cline { 2 - 3 } Phenotype & Spring 2013 & Fall 2013 \\
\hline Green shoulder apple base & $5.00 \mathrm{a}^{\mathrm{z}}$ & $4.21 \mathrm{ab}$ \\
Pale green & $4.73 \mathrm{a}$ & $3.36 \mathrm{c}$ \\
Uniform green & $4.92 \mathrm{a}$ & $3.75 \mathrm{bc}$ \\
Green shoulder & $4.87 \mathrm{a}$ & $3.80 \mathrm{bc}$ \\
Apple green & $4.81 \mathrm{a}$ & $4.21 \mathrm{ab}$ \\
Medium green & $-\mathrm{y}$ & $3.85 \mathrm{ab}$ \\
Medium-green apple base & - & $4.27 \mathrm{a}$
\end{tabular}

$\overline{\mathrm{z}}$ Mean separation in columns by Duncan's multiple range test at $P \leq$ 0.05 .

${ }^{\mathrm{y}}$ No individuals for that phenotypic class.

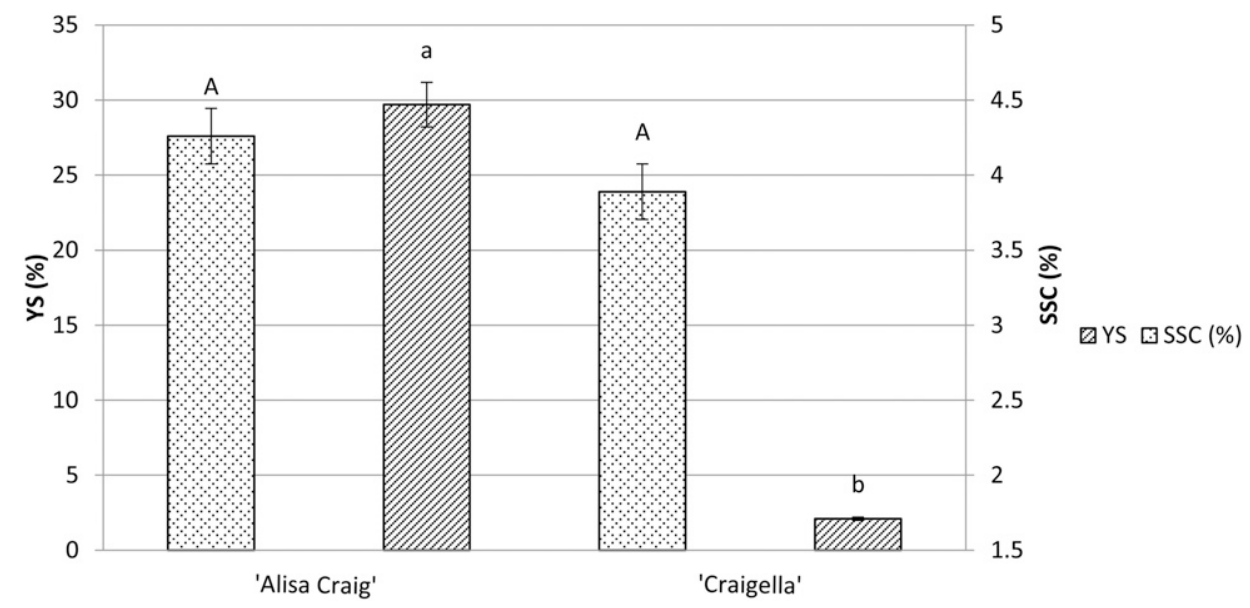

Fig. 3. Incidence of yellow shoulder (YS) and soluble solids concentration (SSC) for 'Alisa Craig' and 'Craigella' tomato cultivars in Fall 2013. Cultivars were compared with a one-way analysis of variance; mean comparisons were made between columns with the same letter case. indicator in these studies because other genotypes had normal green foliage. The sun can bleach out some fruit of uniform green plants to make them look pale green. Thus, the need to carefully observe many fruit per plant for accurate phenotyping.

Soluble solids. Two recent studies found that greenshouldered fruit of 'Ailsa Craig' had higher SSC than that of its isogenic $u$ counterpart cultivar 'Craigella' (Nguyen et al., 2014; Powell et al., 2012). However, when these two cultivars were tested in the field in Fall 2013, the difference, although in the same direction, was not significant (Fig. 3). It is not clear why the results differed between the previous studies and ours. It could be purely an environmental issue related to growing in different locations and/or plants in the previous studies were grown in pots in greenhouses, whereas our plants were grown in the field. It could be that fruit in the previous studies sampled only sun-exposed fruit, and there may have been some canopy cover in our experiment. Another explanation relates to a recent observation (J.W. Scott and S.F. Hutton, unpublished) where plants with medium-green shoulder died from infection with bacterial wilt (Ralstonia solanacearum), and the fruit shoulders of unripe fruit turned dark green similar to that of greenshoulder phenotypes. This would seem to be a stress response of fruit to produce chlorophyll and presumably photosynthesize more when their leaves died and stopped translocating photoassimilates. Possibly, stress plays some role in increasing not only the pigment formation but also the SSC derived from the fruit pigments. If the plants grown in pots in the two greenhouse studies (Nguyen et al., 2014; Powell et al., 2012) were subjected to more stress due to root constriction and/or uneven watering or fertilization, this could have resulted in the significant differences observed between $u^{+}$and $u$. Verification of this hypothesis would require further experimentation.

Regardless, if $u^{+}$enhances SSC because of the darker shoulder, such an effect would be reduced in fruit with partial green shoulder where leaves cover part of the tomato shoulder or eliminated in canopy-covered fruit where the green shoulder does not express. However, beyond this, we found no evidence that green-shoulder genotypes segregating in $18 \mathrm{~F}_{2}$ populations over two seasons had increased SSC over $u$, ug, or other genotypes studied (Table 5). If there is an enhancement of SSC from $u^{+}$over uniform green, uniform gray-green, or other genotypes then it would appear to be contingent on specific environmental conditions and/or genetic backgrounds. There is considerable literature on the role of leaves and fruit in providing photoassimilates to tomato fruit that has been reviewed by Cocaliadis et al. (2014). An indepth discussion of this topic is beyond the scope of this article. However, our results are in line with previous research that indicates fruit are sinks for photosynthetic activity and that leaves provide most photoassimilates to fruit while the fruit themselves are only small contributors (Do et al., 2010; Hackel et al., 2006; Schauer et al., 2006; Zanor et al., 2009).

These results strongly indicate that the fruit color phenotypes 
Table 7. Incidence of yellow shoulder (YS), and immature tomato fruit phenotypes for parents and $\mathrm{F}_{1} \mathrm{~s}$ for Spring and Fall 2013 seasons and the two seasons combined.

\begin{tabular}{|c|c|c|c|c|}
\hline Pedigree & Phenotype & Spring YS (\%) & Fall YS (\%) & Avg YS (\%) \\
\hline Fla. 8033 & Medium green & $54.6 \mathrm{a}^{\mathrm{z}}$ & $36.3 \mathrm{ab}$ & $45.5 \mathrm{ab}$ \\
\hline LA3035 × LA0021 & Green shoulder & $41.2 \mathrm{a}$ & $35.5 \mathrm{ab}$ & $38.4 \mathrm{ab}$ \\
\hline LA0021 × 'Walter' & Green shoulder & $34.3 \mathrm{ab}$ & $33.9 \mathrm{ab}$ & $34.1 \mathrm{ab}$ \\
\hline LA0021 × NC 84173 & Green shoulder & $29.4 \mathrm{ab}$ & $26.3 \mathrm{a}-\mathrm{c}$ & $27.9 \mathrm{ab}$ \\
\hline Fla. 8033 × LA0021 & Medium green & $28.7 \mathrm{a}-\mathrm{c}$ & $21.9 \mathrm{a}-\mathrm{c}$ & $25.3 \mathrm{ab}$ \\
\hline Fla. $8033 \times$ LA3035 & Medium green & $26.4 \mathrm{a}-\mathrm{c}$ & $22.1 \mathrm{a}-\mathrm{c}$ & $24.3 \mathrm{ab}$ \\
\hline LA0021 & Uniform grey-green & $23 \mathrm{a}-\mathrm{c}$ & $20.4 \mathrm{a}-\mathrm{c}$ & $21.7 \mathrm{ab}$ \\
\hline LA0021 × Fla. 7956 & Apple green & $18.8 \mathrm{a}-\mathrm{d}$ & $17.7 \mathrm{a}-\mathrm{c}$ & $18.3 \mathrm{ab}$ \\
\hline 'Walter' $\times$ 'Micro-Tom' & Green shoulder & $20 a-d$ & $14.5 \mathrm{a}-\mathrm{c}$ & $17.3 \mathrm{ab}$ \\
\hline 'Walter' × Fla. 7956 & Green shoulder/apple base & $20.6 \mathrm{a}-\mathrm{d}$ & $13 \mathrm{bc}$ & $16.8 \mathrm{ab}$ \\
\hline LA3035 × 'Walter' & Green shoulder & $17.1 \mathrm{a}-\mathrm{d}$ & $15.5 \mathrm{a}-\mathrm{c}$ & $16.3 \mathrm{ab}$ \\
\hline Fla. $8033 \times$ NC 84173 & Medium green & $18.3 \mathrm{a}-\mathrm{d}$ & $14 \mathrm{bc}$ & $16.2 \mathrm{ab}$ \\
\hline NC 84173 & Uniform green & $16.3 \mathrm{a}-\mathrm{d}$ & $12.2 \mathrm{bc}$ & $14.3 \mathrm{ab}$ \\
\hline LA3035 × NC 84173 & Uniform green & $14.7 \mathrm{a}-\mathrm{d}$ & $13.5 \mathrm{bc}$ & $14.1 \mathrm{ab}$ \\
\hline LA3035 & Uniform green & $20.5 \mathrm{a}-\mathrm{d}$ & $5.6 \mathrm{c}$ & $13.1 \mathrm{ab}$ \\
\hline Fla. $8033 \times$ 'Micro-Tom' & Medium green & $15 \mathrm{a}-\mathrm{d}$ & $9 \mathrm{bc}$ & $12.0 \mathrm{ab}$ \\
\hline 'Walter' & Green shoulder & $19.7 \mathrm{a}-\mathrm{d}$ & $5.2 \mathrm{c}$ & $12.5 \mathrm{ab}$ \\
\hline 'Walter' $\times$ NC 84173 & Green shoulder & $12.7 \mathrm{~b}-\mathrm{d}$ & $11.4 \mathrm{bc}$ & $12.1 \mathrm{ab}$ \\
\hline 'Micro-Tom’' & Pale green & $10 \mathrm{~b}-\mathrm{d}$ & $8 \mathrm{bc}$ & $9.0 \mathrm{~b}$ \\
\hline LA3035 × Fla. 7956 & Apple green & $7.2 \mathrm{~b}-\mathrm{d}$ & $4.3 \mathrm{c}$ & $5.8 \mathrm{~b}$ \\
\hline LA3035 × 'Micro-Tom' & Pale green & $6.3 \mathrm{~b}-\mathrm{d}$ & $4.2 \mathrm{c}$ & $5.3 \mathrm{~b}$ \\
\hline LA0021 × 'Micro-Tom' & Pale green & $5 b-d$ & $4.3 \mathrm{c}$ & $4.7 \mathrm{~b}$ \\
\hline Fla. 7956 & Apple green & $0 \mathrm{~d}$ & $0 \mathrm{c}$ & $0 \mathrm{~b}$ \\
\hline Fla. $7956 \times$ NC 84173 & Apple green & $0 \mathrm{~d}$ & $0 \mathrm{c}$ & $0 \mathrm{~b}$ \\
\hline Fla. $7956 \times$ 'Micro-Tom' & Uniform green & $0 \mathrm{~d}$ & $0 \mathrm{c}$ & $0 \mathrm{~b}$ \\
\hline Fla. $8033 \times$ Fla. 7956 & Apple green & $0 \mathrm{~d}$ & $0 \mathrm{c}$ & $0 \mathrm{~b}$ \\
\hline
\end{tabular}

${ }^{\mathrm{z}}$ Mean separation in columns by Duncan's multiple range test at $P \leq 0.05$. Data were transformed to the arcsin for analysis.

Table 8. Incidence of yellow shoulder (YS) for tomato $F_{2}$ plants grouped by phenotype for Spring and Fall 2013 seasons.

\begin{tabular}{lcr}
\hline & \multicolumn{2}{c}{ YS (\%) } \\
\cline { 2 - 3 } Phenotype & Spring & Fall \\
\hline Green shoulder & $24.2 \mathrm{a}^{\mathrm{z}}$ & $29.9 \mathrm{ab}$ \\
Green shoulder apple base & $15.8 \mathrm{ab}$ & $17.9 \mathrm{~b}$ \\
Uniform green & $10.3 \mathrm{~b}$ & $11.5 \mathrm{~b}$ \\
Apple green & $8.8 \mathrm{~b}$ & $5.8 \mathrm{~b}$ \\
Pale green & $6.5 \mathrm{~b}$ & $14.9 \mathrm{~b}$ \\
Medium green & $-\mathrm{y}$ & $46.7 \mathrm{a}$ \\
Medium green shoulder apple base & - & $6.7 \mathrm{~b}$ \\
\hline
\end{tabular}

${ }^{\mathrm{z}}$ Mean separation in columns by Duncan's multiple range test at $P \leq$ 0.05 . Data were transformed to the arcsin for analysis.

${ }^{\mathrm{y}}$ No individuals planted.

studied are not related to SSC and that the selection of any of them would not have a significant effect on fruit quality associated with SSC. The plants used in this study encompass a wide range of variation typical of that seen in breeding programs. The individuals in the present study differed in many traits in addition to fruit color such as canopy density, plant architecture, flowering time, and leaf morphology. Some traits such as dense canopies in tomato have been shown to increase photoassimilate levels in fruit (Ho, 1980). Importantly, we found no evidence that tomato breeders should select for green shoulders to enhance SSC.

The reported enhancement of SSC from $u^{+}$could have been an effect of this specific gene or a product of the increased chlorophyll content as hypothesized by Powell et al. (2012). In the latter case, the apple green genotype would also be expected to have increased SSC, whereas pale green genotypes would be expected to have lower SSC. Yet no support for fruit darkness having an effect on SSC was found. First of all, the SSC for parents and $F_{1} s$ was higher in the spring than the fall despite the fruit being darker in the fall (Tables 2 and 5). These seasonal fruit color differences are supported by Smillie et al. (1999) who found that green fruit pigmentation was suppressed with high levels of light. Regression analyses revealed nonsignificant correlation coefficients of 0.06 and 0.08 for fruit darkness L scores and SSC in the spring and fall, respectively. In Fall 2013, there was a trend for $F_{2}$ apple green segregants to have higher SSC (Table 5), but because apple green has darker green foliage as well as fruit, the increased SSC could have resulted from the increased chlorophyll in the fruit, the foliage, or both. Fruit size has not been mentioned, but if $u^{+}$has an effect on SSC, then such an effect would be more likely observed with small-fruited genotypes such as 'Ailsa Craig' because the surface to volume ratio is larger than for a large-fruited genotype such as 'Walter'. We intended to partition $\mathrm{F}_{2}$ plants by fruit size to see if it was a factor in $u^{+} \mathrm{SSC}$, but most of the fruit in this study were considered to be small fruited, so there was no reason to do this. Significantly higher SSC was seen in the spring. A similar result was reported by Georgelis et al. (2006), who measured tomato SSC in Florida under similar conditions. Temperature conditions for growing tomatoes were favorable in both fall and spring seasons. In Spring 2013, rainfall was higher, particularly in June when SSC measurements were made. Generally, increased irrigation rates have a negative impact on SSC (May et al., 1990). Increased rainfall during fruit ripening would have been expected to decrease SSC, especially the $3.96 \mathrm{~cm}$ of rainfall on 20 June, $1 \mathrm{~d}$ before SSC measurements began. However, spring results showed an increase in SSC despite increased rainfall. A possible explanation for higher spring sugar levels was the higher solar radiation resulting in elevated photosynthetic rates, thereby producing more carbohydrates, which is in agreement with previous studies (Davies and Hobson, 1981; Georgelis et al., 2006). The temperature in the spring was higher; however, the effect of temperature on soluble solids levels in tomato is unknown. Further research is needed to understand the possible interactions between SSC, temperature, and solar radiation.

Yellow Shoulder. Picha and Hall (1981) found that a uniform green-shouldered cultivar Healani had a high level of resistance to YS, whereas three cultivars with green shoulder were susceptible. Many have observed that green shoulder 
genotypes are more prone to increased YS incidence than uniform green genotypes (Picha, 1987; Picha and Hall, 1981; Scott, 2014; Venter, 1965; Yeager, 1935). However, we report the first definitive data to actually demonstrate this premise. YS incidence was significantly greater in $\mathrm{F}_{2}$ green-shoulder segregants than in uniform-shoulder segregants that did not differ from each other in Spring 2013 (Table 8). In the fall, mediumgreen-shouldered $\mathrm{F}_{2}$ segregants had significantly more YS than any of the uniform-green phenotypes but did not differ from segregants with green-shouldered fruit, and the latter was not significantly different from the uniform shoulder phenotypes. Because the uniform shoulder types included the relatively dark-colored apple green and the relatively light-colored pale green, it would seem that darkness of pigment per se had little to do with YS incidence (Tables 3 and 8). In addition, the other genotype that was similar to green shoulder in YS susceptibility was medium green, although medium-green fruit shoulders were lighter green than for $u^{+}$genotypes. The genetic relationship between green shoulder and medium-green shoulder is unknown because a cross between the two failed (Mattia and Scott, 2014). However, in that study, segregation ratios of medium green with other genotypes studied here behaved similar to those of green shoulder. This suggests that medium green may be an alternative allele to $u^{+}$but with a reduced production of chlorophyll in the fruit shoulder. Regardless, Romero-Aranda et al. (2004) showed that temperature alone did not cause YS, but that solar radiation and/or light quality played an essential role in YS expression. Putting this together, it may be that solar radiation interacts more readily with green shoulder and medium-green shoulder genetic mechanisms to induce a breakdown in chromoplast formation that then reduces cell size (Francis et al., 2000) and subsequent carotenoid production resulting in yellow color. Apparently, the genetic mechanism of chlorophyll production in apple green fruit is different from that of green shoulder and medium-green shoulder and not as prone to YS damage even though fruit are a darker green. Obviously, more studies are needed to verify these suppositions.

The lack of significant differences in YS incidence between any of the uniform immature fruit color phenotypes (apple green, pale green, and uniform green) can be used in breeding to significantly decrease YS despite their differences in color. This is especially interesting in the case of apple green because it was believed that YS incidence was often greater in greenshoulder genotypes because the darker shoulder pigment absorbs more heat from the sun. Yet, in the spring, apple green $\mathrm{F}_{2} \mathrm{~S}$ were darker than green shoulder and medium-green $\mathrm{F}_{2}$ plants, and in the fall, apple green $F_{2}$ fruit color was intermediate to green shoulder and medium-green shoulder (Table 3). Perhaps it is the difference in shoulder and base colors that is more critical to YS incidence.

'Walter', the green-shouldered cultivar used in this experiment, was reported to be resistant to YS (Strobel el al., 1969), and our results showed that 'Walter' had intermediate resistance to $\mathrm{YS}$. However, despite this resistance, in $\mathrm{F}_{2} \mathrm{~S}$ mostly derived from 'Walter' crosses, segregating plants with green shoulder had significantly more YS than phenotypes with uniform shoulder and base colored fruit in the spring, but not in the fall experiment. The data also show that selecting plants that have uniform ripening will not completely eliminate the problem of YS because there was some YS seen in all immature green phenotypes. Fla. 7956 appeared to be totally resistant to YS and could be useful for breeding or other studies regarding YS.

\section{Conclusions}

It was suggested by Powell et al. (2012) that tomato breeders, in selecting for uniform fruit shoulders, had inadvertently reduced SSC and thus reduced tomato fruit quality of both fresh and processed tomato cultivars developed over the last several decades. This was based on the comparison of two isogenic cultivars with differences in fruit shoulder color where the green-shouldered one had higher SSC than the uniformshouldered one. Herein, we tested a more diverse set of lines and made crosses to compare immature fruit color genes in segregating populations. Our data suggest that shoulder color does not play a significant role in the SSC of the ripe fruit. Therefore, we cannot support the concept that the selection for uniform green-shouldered fruit had a significant effect on soluble solids and the flavor of tomato cultivars. Rather our data support literature that suggests that fruit SSC is mainly translocated from the leaves of the plant. However, we do hypothesize that immature fruit color might have a greater role in SSC when plants are stressed; this hypothesis would have to be tested, however, because we only speculate on this possibility. We did provide data for the first time that showed that plants with green-shouldered fruit and those with a lesserknown phenotype, medium-green shoulder, had more YS than phenotypes with uniform shoulders. Moreover, the darkness of green in the fruit shoulders per se did not cause increased YS.

\section{Literature Cited}

Bohn, G. and D.H. Scott. 1945. A second gene for uniform unripe fruit color in the tomato. J. Hered. 36:169-172.

Cocaliadis, M.F., R. Fernandez-Munoz, C. Pons, D. Orzaez, and A. Granell. 2014. Increasing tomato fruit quality by enhancing fruit chloroplast function. A double-edged sword? J. Expt. Bot. 65:4589-4598.

Davies, J.N., G.E. Hobson, and W.B. McGlasson. 1981. The constituents of tomato fruit-The influence of environment, nutrition, and genotype. Crit. Rev. Food Sci. Nutr. 15:205-280.

Do, P.T., M. Prudent, R. Sulpice, M. Causse, and A.R. Fernie. 2010. The influence of fruit load on the tomato pericarp metabolome in a Solanum chmielewskii introgression line population. Plant Physiol. 154:1128-1142.

Francis, D.M., S.A. Barringer, and R.E. Whitmoyer. 2000. Ultrastructural characterization of yellow shoulder in a uniform ripening tomato genotype. HortScience 35:1114-1117.

Georgelis, N., J.W. Scott, and E.A. Baldwin. 2006. Inheritance of high sugars from tomato accession PI 270248 and environmental variation between seasons. J. Amer. Soc. Hort. Sci. 131:41-45.

Hackel, A., N. Schauer, F. Carrari, A.R. Fernie, B. Grimm, and C. Kühn. 2006. Sucrose transporter LeSUT1 and LeSUT2 inhibition affects tomato fruit development in different ways. Plant J. 45:180-192.

Ho, L.C. 1980. Control of import into tomato fruits. Plant Biol. 93:315-325.

Kemp, G.A. and I.L. Nonnecke. 1960. Differences in intensity of unripe fruit colour in the tomato. Can. J. Plant Sci. 40:306-309.

Mattia, M.R. and J.W. Scott. 2014. Apple green and pale green fruit colors are controlled by alleles at the uniform green $(u)$ locus on chromosome 10. Rpt. Tomato Genet. Coop. 64:25-30.

May, D.M., D. Peters, D. Wolcott, and D.W. Grimes. 1990. Moisture stress as it affects yield, soluble solids and viscosity of tomatoes. Acta Hort. 277:123-128.

Nguyen, C.V., J.T. Vrebalov, N.E. Gapper, Y. Zheng, S. Zhong, Z. Fei, and J.J. Giovannoni. 2014. Tomato GOLDEN2-LIKE transcription factors reveal molecular gradients that function during fruit development and ripening. Plant Cell Online 26:585-601.

Olson, S.M., W.M. Stall, G.E. Vallad, S.E. Webb, S.A. Smith, E.H. Simonne, E. McAvoy, B.M. Santos, and M. Ozores-Hampton. 2011. 
Tomato production in Florida, p. 409-428. In: S.M. Olson and B.M. Santos (eds.). Vegetable production handbook for Florida. Inst. Food Agr. Sci., Univ. Florida, Gainesville, FL.

Picha, D.H. 1986. Effect of harvest maturity on the final fruit composition of cherry and large-fruited tomato cultivars. J. Amer. Soc. Hort. Sci. 111:723-727.

Picha, D.H. 1987. Physiological factors associated with yellow shoulder expression in tomato fruit. J. Amer. Soc. Hort. Sci. 112:798-801.

Picha, D.H. and C.B. Hall. 1981. Influences of potassium, cultivar, and season on tomato graywall and blotchy ripening. J. Amer. Soc. Hort. Sci. 106:704-708.

Powell, A.L., C.V. Nguyen, T. Hill, K.L. Cheng, R. Figueroa-Balderas, H. Aktas, H. Ashrafi, C. Pons, R. Fernández-Muñoz, A. Vicente, and J. Lopez-Baltazar. 2012. Uniform ripening encodes a Golden 2-like transcription factor regulating tomato fruit chloroplast development. Science 336:1711-1715.

Reynard, G.B. 1952. Genes for uniform ripening of fruits. Rpt. Tomato Genet. Coop. 2:7.

Reynard, G.B. 1956. Origin of the Webb Special (Black Queen) tomato. Rpt. Tomato Genet. Coop. 6:22.

Reynard, G.B. 1960. Levels of pigment intensity of immature fruits. Rpt. Tomato Genet. Coop. 10:28-29.

Romero-Aranda, R., R. Fernández-Muñoz, G. López-Casado, and J. Cuartero. 2004. Yellow shoulder in tomatoes under natural and controlled conditions. TGC Rpt. 54:34-35.
Schauer, N., Y. Semel, U. Roessner, A. Gur, I. Balbo, F. Carrari, T. Pleban, A. Perez-Melis, C. Bruedigam, J. Kopka, and L. Willmitzer. 2006. Comprehensive metabolic profiling and phenotyping of interspecific introgression lines for tomato improvement. Nature Biotechnol. 24:447.

Scott, J.W. 2014. Yellow shoulder, p. 134. In: Compendium of tomato diseases, 2nd ed. APS Press, St. Paul, MN.

Smillie, R.M., S.E. Hetherington, and W.J. Davies. 1999. Photosynthetic activity of the calyx, green shoulder, pericarp, and locular parenchyma of tomato fruit. J. Expt. Bot. 50:707-718.

Strobel, J.W., N.C. Hayslip, D.S. Burgis, and P.H. Everett. 1969. Walter: A determinate tomato resistant to races 1 and 2 of the fusarium wilt pathogen. Florida Agr. Exp. Sta. Circ. S.

Venter, F. 1965. Investigation on green-back of tomatoes, p. 99-101. In: Symposium on Vegetable Growing under Glass. Intl. Soc. Hort. Sci., The Hague, The Netherlands.

Yeager, T. 1935. The uniform fruit color gene in the tomato. Proc. Amer. Soc. Hort. Sci. 33:512.

Zanor, M.I., S. Osorio, A. Nunes-Nesi, F. Carrari, M. Lohse, B. Usadel, and A.R. Fernie. 2009. RNA interference of LIN5 in tomato confirms its role in controlling soluble solids concentration, uncovers the influence of sugars on the levels of fruit hormones, and demonstrates the importance of sucrose cleavage for normal fruit development and fertility. Plant Physiol. 150:1204-1218. 